\title{
LA REALTÀ FISICA, MON BEAU SOUCI
}

\author{
PAOLO PERINOTTI (*)
}

SuNTO. - Nel contributo si espone il personale punto di vista di un fisico teorico sul legame tra realtà e scienza fisica, partendo dalla propria esperienza nell'ambito dei fondamenti della meccanica quantistica e della teoria quantistica dell'informazione. Si sostiene l'efficacia di un approccio assiomatico rigoroso come sistema di navigazione per muoversi tra un paesaggio astratto, quello quantistico, e la realtà fattuale, lungo un percorso costitutivamente sfuggente ai classici sistemi di orientamento. Tale approccio richiede alla fisica di accogliere al suo livello più fondamentale l'informazione in sostituzione alla materia ed allo spazio-tempo, i quali riemergono ad un secondo livello, come descrizioni efficaci. L'esito di tale operazione è un panorama concettuale semplificato, in cui nuove possibilità si aprono per affrontare le sfide aperte della fisica teorica.

ABSTRACT. - The personal viewpoint of a theoretical physicist about the relation between reality and physical science is exposed, starting from his personal experience in the context of quantum foundations and quantum information theory. The effectiveness of an axiomatic approach is argued as a navigation system between the abstract landscape of quantum theory and the reality of facts, along a road that is intrinsically unaccessible to classical conceptual maps. The present approach requires physics to accept the notion of information at its deepest level, in place of matter and space-time, that are recovered only as an effective description of phenomena on a secondary level. As a result of the above operation, a simplified conceptual scenario is achieved, where new possibilities are available for facing the open challenges of theoretical physics.

Questo contributo si propone di rappresentare il personale punto di vista di un fisico teorico sul legame tra realtà e scienza fisica, partendo dalla propria esperienza nell'ambito dei fondamenti della meccanica quantistica e della teoria quantistica dell'informazione. La rivoluzione della fisica avvenuta nei primi decenni del XX secolo ha creato un

(*) Università degli Studi di Pavia, Italia. E-mail: paolo.perinotti@unipv.it 
ampio solco tra il paesaggio astratto della teoria quantistica e la realtà fattuale, lasciando scoperta una ampia zona in cui hanno preso vita le tante interpretazioni della teoria, zona che invece era apparentemente assente nel precedente contesto della fisica classica. In tale zona trova senso il presente contributo, a partire dal suo titolo.

Esso parafrasa quello di un articolo del 1965 del regista Jean Luc Godard, "Montage, mon beau souci", pubblicato sulla rivista "Cahiers du Cinema", in cui l'autore interviene nell'ambito di in un dibattito sull'estetica nel cinema. Lo scritto di Godard rappresenta la risposta alla tesi sostenuta da molti registi del tempo, ovvero che il montaggio debba sparire al cospetto della sceneggiatura. L'autore reagisce sostenendo l'importanza del montaggio nella costruzione di un film, restituendogli un ruolo centrale nell'arte cinematografica accanto alla sceneggiatura. Io ho preso in prestito questo dibattito come metafora per simboleggiare da un lato le posizioni di chi sostiene che l'osservazione della realtà sia da paragonarsi alla ripresa fedele di una sceneggiatura, e dall'altro quelle di chi ritiene che l'osservatore abbia un ruolo attivo nella formazione della realtà, consistente nel suo modo di raccogliere e dare ordine agli eventi, in un'operazione accostabile al montaggio nel cinema. Ciò detto, è anche evidente come io mi schieri tra i fautori della seconda posizione.

La prospettiva che propongo deriva da un modo di interpretare la meccanica quantistica, che più precisamente consiste nell'interpretarla il meno possibile. Tale approccio si colloca nel solco delle posizioni che furono di Niels Bohr [1], e più tardi nel corso del XX secolo del fisico statunitense John Archibald Wheeler [2].

Uno degli aspetti più famosi della meccanica quantistica è il principio di indeterminazione [3], che sancisce l'esistenza di grandezze complementari, le quali non possono assumere simultaneamente valori definiti. Si potrebbe essere tentati di pensare che tale limitazione, intesa come divieto ontologico, sia eccessiva, e che l'indeterminazione quantistica rappresenti solamente un vincolo epistemico, dovuto ad esempio ai sistemi di misura che utilizziamo, mentre in realtà tutte le grandezze di un sistema fisico abbiano sempre valori ben definiti. Tale idea tracciò la linea di pensiero di Einstein a proposito dell'indeterminazione quantistica, e lo spinse, insieme a Podolski e Rosen, a proporre nel 1935 un esperimento ideale [4] che nelle intenzioni degli autori doveva mettere in crisi la completezza della meccanica quantistica, riducendola ad una sorta di teoria statistica, efficace ma non fondamentale. Tuttavia, i fatti 
che seguirono quella proposta ci dicono che la situazione è più complessa di come la immaginava Einstein.

L'argomento chiave da cui parte la mia riflessione è infatti la violazione della disuguaglianza di Bell [5]. Tale disuguaglianza riguarda le correlazioni tra le grandezze fisiche misurate in esperimenti del tipo di quello proposto da Einstein, Podolski e Rosen. Questi esperimenti coinvolgono sistemi composti di due o più parti, ad esempio una coppia di elettroni, e si svolgono a grandi linee come segue: i due elettroni si allontanano dalla sorgente e raggiungono due laboratori distanti tra loro. Nei due laboratori ci sono due fisici, che chiameremo Alice e Bob. Entrambi possono scegliere tra due grandezze complementari da misurare sul proprio elettrone, tipicamente due componenti dello spin.

Se il sistema dei due spin si trova in un particolare tipo di stato, detto "entangled", l'esperimento manifesta correlazioni molto speciali tra le quantità misurate sui due elettroni. La caratteristica peculiare di tali correlazioni è quella di essere impossibili da ottenere se i valori delle grandezze misurate sull'elettrone A di Alice fossero, come ci aspetteremmo, tutti quanti definiti e codificati nell'elettrone $A$, sin da prima dell'atto della misurazione. Questo risultato è noto come violazione delle disuguaglianze di Bell. Ė dagli anni 1980 che si è incominciato a verificare tale violazione negli esperimenti condotti pionieristicamente da Alain Aspect [6], ed in seguito da molti altri scienziati, per arrivare allo scorso biennio in cui esperimenti di nuova generazione, considerati conclusivi perché escludono alcune residue possibilità di interpretazione classica, sono stati portati a termine con successo [7].

Lo scenario sconcertante che tale risultato apre ci lascia tre possibilità per interpretare la statistica quantistica.

La prima si chiama "super-determinismo". Possiamo semplificare il contenuto di questa posizione, dicendo che la natura è architettata maliziosamente in modo tale che tutti gli eventi fisici, ivi incluse le nostre scelte tra quantità complementari da misurare su di un dato sistema, sono pre-determinati e finemente correlati, in modo da darci l'illusione che valga la statistica quantistica. Personalmente, trovo tale posizione altamente anti-scientifica, in quanto, se portata alle estreme conseguenze, essa svuoterebbe la fisica di qualunque valore conoscitivo [8].

La seconda via consiste nel salvare l'idea che la misura sia lettura di un valore pre-esistente, al prezzo però di ammettere che tale valore non venga determinato esclusivamente dallo stato del sistema osservato, ma dal concorso istantaneo di tutti i sistemi dell'universo. Il motivo 
per cui non condivido neppure questa seconda posizione è che essa di fatto asserisca l'inadeguatezza del metodo sperimentale, basato sul meticoloso controllo delle condizioni locali per escludere influenze sull'esperimento da parte di terzi sistemi. Tanto più in quanto gli elementi di realtà non locale che determinerebbero i risultati delle misure, secondo tale interpretazione, sono costituzionalmente inaccessibili alla osservazione sperimentale. Su di un piano esclusivamente epistemologico, inoltre, affinché tale interpretazione sia fisicamente accettabile, gli elementi di realtà devono essere mascherati da qualche meccanismo ad hoc, al fine di non permettere comunicazioni istantanee a distanza.

La terza via resta, a mio parere, la meno problematica. Essa consiste nel pensare il risultato della misura come un'informazione generata dalla relazione tra sistema misurato ed osservatore. Questa posizione chiarisce la ragione del titolo del mio intervento: credo che la realtà degli eventi che osserviamo, essendo una collezione di risultati di misure ed osservazioni, scaturisca dall'interazione tra osservatori e sistemi osservati, e la sua ricostruzione consista in un processo simile al montaggio di informazioni per formare un immaginario quanto più possibile coerente e soprattutto capace di interpretare il manifestarsi di eventi futuri.

Non negherò certamente i problemi filosofici aperti da questa posizione, in primis il ruolo privilegiato svolto dall'osservatore nell'ambito della fisica. Credo, tuttavia, che molte delle difficoltà nel comprendere ed accettare tale visione siano dovute al fatto che, per oltre settant'anni, la formulazione della teoria quantistica sia rimasta esclusivamente matematica, con assiomi astratti di cui è impossibile trovare una diretta traduzione sul piano operativo. Tale situazione sposta l'interpretazione dagli asserti fondamentali della teoria - i postulati - ad un livello molto più indiretto, quello dei sofisticati oggetti matematici che ne discendono: algebre, operatori, funzionali, mappe, e via elencando. Questo spostamento lascia troppo arbitrio nell'interpretazione.

L'esperienza della teoria quantistica dell'informazione che data ad oggi una trentina di anni, ha offerto una nuova prospettiva su fondamenti, fornendo una visione della teoria quantistica sempre più prossima ad una teoria dei portatori di informazione e dei loro processi di elaborazione. Tale visione ha permesso a diversi autori, tra i quali Giulio Chiribella, Giacomo Mauro D'Ariano e l'autore [9], di formulare la teoria mediante assiomi che hanno due livelli di lettura, uno puramente matematico, ed uno fisico, in cui gli assiomi si traducono in divieto o possibilità di effettuare operazioni di elaborazione di informazione. A 
titolo di esempio, richiameremo solamente il primo postulato della citata formulazione, quello di causalità. Esso asserisce che la scelta di quale grandezza misurare su di un sistema non può influenzare la probabilità delle sue diverse possibili preparazioni. In altri termini, l'influenza causale non può fluire nella direzione che va dalla misura verso la preparazione, ma solo in quella opposta.

La nuova formulazione della teoria vede i sistemi quantistici prima facie come "registri di memoria", che codificano informazionitale è l'interpretazione dei loro stati-e non come particelle materiali o modi di vibrazione, come nella tradizione della fisica quantistica. I sistemi quantistici non sono pertanto caratterizzati primariamente quali "costituenti elementari della materia", e diventano piuttosto "supporti elementari di informazione". Gli aspetti fisici dei sistemi sono determinati solamente in seconda istanza, dalle loro modalità di evoluzione ed interazione.

Uno dei punti di forza del nuovo approccio è quello di superare il confronto trito tra teoria classica e teoria quantistica. Tale confronto non può che far apparire la teoria quantistica bizzarra e scomoda, per il semplice fatto che l'altro termine di paragone è la teoria nata per descrivere i fenomeni consueti nella nostra esperienza quotidiana, quali la caduta di un grave, il moto dei corpi celesti o l'urto di due palle da biliardo.

I postulati della nuova formulazione individuano invece la teoria quantistica in un più ampio contesto di possibili teorie dei sistemi, le teorie operazionali probabilistiche, di cui quella quantistica e quella classica non sono che due esempi.

Il primo punto su cui mi soffermerò è l'importanza dell'analisi comparativa tra la teoria quantistica e altre teorie alternative, che non siano quella classica. Ad esempio, se volessimo costruire una teoria generica, saremmo tenuti, tra l'altro, ad indicare le probabilità dei risultati di misure a due valori su due parti di un sistema composto in uno stato puro. Per una specifica coppia di misure, questo equivale a scegliere un punto all'interno del tetraedro indicato nella Fig. 1. La teoria manifesterà entanglement se il punto scelto non cade sulla superficie a sella evidenziata in viola [10].

$\mathrm{Ci}$ si accorge allora che non è tanto l'entanglement ad essere una stravaganza quantistica, quanto la sua assenza ad essere una caratteristica molto specifica della teoria classica. Diventa quindi molto più ragionevole l'accettazione di caratteristiche contro-intuitive della meccanica 
quantistica, in quanto esse sono tipiche, se si ragiona a priori nel panorama delle possibili teorie, mentre diventa meno giustificato il tentativo di salvare le peculiari caratteristiche della teoria classica, quali il realismo delle grandezze fisiche.

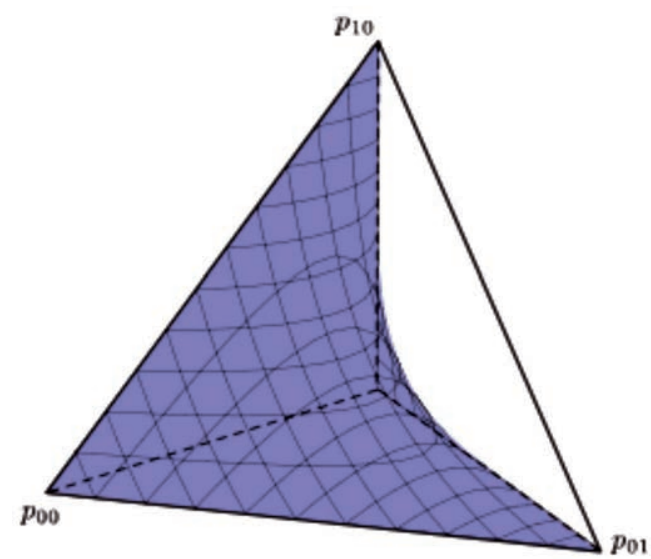

Fig. 1. Il tetraedro indica la collezione dei punti che possono corrispondere alle probabilità dei risultati di due misure effettuate sulle due componenti di un sistema composto. La superficie a sella evidenziata in viola rappresenta la collezione dei punti corrispondenti a probabilità che non danno luogo ad entanglement. La condizione individuata dalla superficie viola è chiaramente molto speciale, escludendo la maggior parte dei punti possibili (in termini tecnici essa individua un insieme di misura nulla).

Il secondo punto che vorrei sottolineare è che, così come la materia, nell'approccio "informazionale" ai fondamenti della meccanica quantistica, e più in generale della fisica, anche lo spazio ed il tempo o lo spazio-tempo, parlando in termini relativistici - non sono elementi primari. Lo spazio-tempo emerge come epifenomeno [11], non in quanto "contenitore" delle leggi fisiche come nella visione di Newton, ma come modo di descrizione compatta ed efficace di queste ultime, che possono però essere formulate astrattamente come algoritmi di elaborazione delle informazioni elementari, indipendentemente dalla geometria. Tale aspetto è di grande rilevanza perché apre nuovi orizzonti ad una teoria che concilii meccanica quantistica e gravità.

Prima di concludere, sento la necessità di fare due osservazioni. La prima riguarda l'apparente conseguenza della posizione che ho 
espresso, ovvero che l'osservatore possa influenzare l'esito della misura. Tale conclusione è profondamente errata. Questo fraintendimento è alla base di tante correnti pseudo-scientifiche da cui desidero prendere esplicitamente le distanze, a scanso di equivoci. Il ruolo dell'osservatore è solamente quello di decidere quale grandezza venga osservata, o in altre parole quale domanda venga posta al sistema, non quale risposta verrà fornita. Dato infatti che le probabilità con cui si ottengono le diverse possibili risposte dipendono solamente dallo stato del sistema, consegue immediatamente dal postulato di causalità che la scelta dell'osservatore, non potendo influenzare tale stato, non può influenzare neppure le probabilità delle risposte. Il valore misurato è quindi totalmente indipendente dalle scelte dell'osservatore.

La seconda osservazione, invece, riguarda l'argomento dovuto a Bell, ovvero che non debbano avere posto in una formulazione della teoria concetti come osservabile, informazione, misura. A questo replico che, per quanto possa concedere che tale situazione sia desiderabile, non ritengo che il fatto di richiederla a priori abbia alcun carattere di necessità. In questo senso proprio la violazione delle disuguaglianze di Bell costituisce uno spartiacque: non si può più escludere a priori che tali concetti facciano parte della formulazione di una teoria, così come dal 1905, anno in cui nacque la teoria della relatività ristretta, non si può più escludere che la simultaneità di due eventi, sino ad allora considerata indiscutibilmente assoluta, sia relativa o addirittura convenzionale.

In conclusione entrerò più direttamente nel tema della relazione tra la realtà ed il realismo. Da scienziato razionalista, la mia tendenza è quella, profondamente scettica, di non accettare a priori nessun asserto, se non supportato da adeguate evidenze. Quando si parla di asserti riguardanti la realtà, pertanto, mi trovo in un terreno che per me è scomodo, perché il verbo principale a cui devo ricorrere è "credere". Accettando le regole del gioco, dirò che credo che esista una realtà a me esterna. Ciò su cui invece mi dichiaro agnostico è se lo scopo della scienza sia di descrivere o spiegare l'essenza di tale realtà esterna, oppure sia di farlo solo in parte, limitandosi ai fenomeni che possiamo osservare. La principale ragione del mio agnosticismo è che anche qualora uno scienziato avesse esposto la teoria "finale", nessuno sarebbe in grado di dimostrare che essa fotografa esattamente "la realtà". Più in generale, non credo neppure che sia necessario che la mente umana possa partorire la teoria "finale", non credo che nessun argomento possa supportare tale convinzione. 
Alla luce di questa posizione agnostica, ci si potrebbe chiedere perché insistere sulla necessità di fondare la fisica su principi e postulati. La mia risposta personale non è: "perché si può fare scienza con soli concetti a priori", ma piuttosto: "perché questo è l'unico modo che abbiamo per formulare una teoria, ed una buona scelta dei principi rende più efficace la comprensione della teoria che da essi scaturisce, e facilita il progresso verso nuove teorie". Per esemplificare questa affermazione, basti pensare alla differenza tra il principio di causalità enunciato prima, ed il postulato della formulazione standard della teoria quantistica che afferma "ogni grandezza osservabile è associata ad un operatore autoaggiunto". È facile immaginare le conseguenze della rimozione del principio di causalitàad esempio la possibilità di comunicare informazioni verso il passato. $\mathrm{Al}$ contrario, se supponiamo di sostituire il postulato che le grandezze osservabili corrispondano ad operatori autoaggiunti con la richiesta, ad esempio, che esse corrispondano ad operatori simmetrici, la comprensione delle conseguenze sulle predizioni della teoria richiedono un lungo sviluppo di teoremi e calcoli matematici. Ciò implica un molto minore controllo sulle modalità di modifica della teoria nel caso di una formulazione priva di interpretazione operazionale, ed una struttura formale necessariamente più rigida.

\section{BIBLIOGRAFIA}

[1] N. Bohr, "Essays 1932-1957 on Atomic Physics and Human Knowledge", ristampato con il titolo "The Philosophical Writings of Niels Bohr", vol. II (Ox Bow Press, Woodbridge, 1987).

[2] J.A. Wheeler, "Information, physics, quantum: The search for links", in "Complexity, Entropy, and the Physics of Information", ed. W. H. Zurek, (Addison-Wesley, Redwood City, 1990).

[3] W. Heisenberg, "Über den anschaulichen Inhalt der quantentheoretischen Kinematik und Mechanik", Zeitschrift für Physik 43, 172-198 (1927).

[4] A. Einstein, B. Podolsky, N, Rosen, "Can Quantum-Mechanical Description of Physical Reality be Considered Complete?", Physical Review 47, 777-780 (1935).

[5] A scopo divulgativo, cito qui il famoso risultato di Bell [J. Bell, "On the EinsteinPoldolsky-Rosen paradox", Physics 1, 195-200 (1964)], anche se il mio discorso fa più precisamente riferimento ad un altro argomento, dovuto a Clauser, Horne, Shimony e Holt [J. F. Clauser, M. A. Horne, A. Shimony, R. A. Holt, "Proposed experiment to test local hidden-variable theories", Physical Review Letters 23, 880-884 (1969)]. 
[6] A. Aspect, P. Grangier, and G. Roger, "Experimental Tests of Realistic Local Theories via Bell's Theorem”, Physical Review Letters 47, 460 (1981).

[7] B. Hensen et. al., "Loophole-free Bell inequality violation using electron spins separated by 1.3 kilometres", Nature 526 (2015), 682-686; M. Giustina et al., "Significant-Loophole-Free Test of Bell's Theorem with Entangled Photons", Phys. Rev. Lett. 115 (2015), 250401; L. K. Shalm et al., "Strong Loophole-Free Test of Local Realism” Phys. Rev. Lett. 115 (2015), 250402.

[8] Il super-determinismo si riduce infatti a due possibilità: o le leggi della fisica sono un inganno sottilmente perpetrato dalla natura, che viene mantenuto eternamente-e allora il super-determinismo sarebbe un inutile fardello ideologico, risultando dal punto di vista predittivo del tutto equivalente ad altre interpretazioni-oppure prima o poi l'inganno viene svelato, ovvero si verificano eventi che contraddicono qualunque legge fisica. In questo secondo caso, tuttavia, le leggi fisiche non avrebbero alcun valore.

[9] G. Chiribella, G. M. D'Ariano and P. Perinotti, "Informational derivation of quantum theory”, Physical Review A 84 (2011), 12311; G. Chiribella, G. M. D'Ariano, and P. Perinotti, "Probabilistic theories with purification", Physical Review A 81 (2010), 62348.

[10] G.M. D'Ariano, F. Manessi, and P. Perinotti, "Spooky action-at-a-distance in general probabilistic theories”, Physics Letters A 376 (2012), 2926.

[11] G.M. D'Ariano, and P. Perinotti, "Derivation of the Dirac equation from principles of information processing”, Physical Review A 90 (2014), 062106. 